\title{
Anisotropic displacement parameters for hydrogen atoms using an ONIOM approach
}

\author{
Andrew E. Whitten ${ }^{\text {ał }}$ and Mark A. Spackman ${ }^{\mathrm{b}^{*}}$ \\ ${ }^{a}$ Chemistry, University of New England, Armidale NSW 2351, Australia, and \\ ${ }^{b}$ Chemistry - M313, University of Western Australia, Crawley WA 6009, Australia. E- \\ mail:mas@cyllene.uwa.edu.au \\ $\ddagger$ Present address: Bragg Institute, Australian Nuclear Science and Technology Organisation, \\ PMB 1, Menai NSW 2234, Australia.
}

Synopsis Ab initio ONIOM cluster calculations are combined with TLS analysis of heavy atom ADPs from X-ray diffraction data to provide accurate estimates of hydrogen atom ADPs for charge density analysis.

Abstract X-ray diffraction data cannot provide anisotropic displacement parameters (ADPs) for hydrogen atoms, a major outstanding problem in charge density analysis of molecular crystals. Although neutron diffraction experiments are the preferred source of this information, for a variety of reasons they are possible only for a minority of materials of interest. To date, approximate procedures combine rigid body analysis of the molecular heavy atom skeleton, based on ADPs derived from the X-ray data, with estimates of internal motion provided by spectroscopic data, analyses of neutron diffraction data on related compounds, or ab initio calculations on isolated molecules. Building on these efforts, we present an improved methodology, incorporating information on internal vibrational motion from ab initio cluster calculations using the ONIOM approach implemented in Gaussian03. The method is tested by comparing model hydrogen atom ADPs with reference values, largely from neutron diffraction experiments, for a variety of molecular crystals: benzene, 1methyluracil, $\alpha$-glycine, xylitol and 2-methyl-4-nitroaniline. The results are impressive, and as the method is based on widely available software, and is in principle widely applicable, it offers considerable promise in future charge density studies of molecular crystals.

Keywords: anisotropic displacement parameters; hydrogen atoms; cluster calculations; charge density analysis; thermal motion 


\section{Supplementary material. TLS+ONIOM ADPs for $\mathrm{H}$ atoms (in units of $10^{-2} \AA^{2}$ )}

Table S1 1-methyluracil @ 21 K. First row: TLS+ONIOM results; second row: adjusted neutron reference results (McMullan \& Craven, 1989); third row: differences.

\begin{tabular}{lccccccc}
\hline & & $U_{11}$ & $U_{22}$ & $U_{33}$ & $U_{12}$ & $U_{13}$ & $U_{23}$ \\
\hline H3 & ref. & 1.56 & 1.06 & 2.53 & -0.30 & - & - \\
& calc. & 1.58 & 1.07 & 2.65 & -0.38 & - & - \\
& $\Delta$ & -0.02 & -0.01 & -0.12 & 0.07 & - & - \\
H5 & ref. & 1.99 & 1.17 & 3.42 & 0.34 & - & - \\
& calc. & 1.88 & 1.08 & 3.27 & 0.38 & - & - \\
& $\Delta$ & 0.11 & 0.09 & 0.16 & -0.05 & - & - \\
H6 & ref. & 1.60 & 1.20 & 2.84 & -0.53 & - & - \\
& calc. & 1.61 & 1.23 & 3.32 & -0.48 & - & - \\
& $\Delta$ & -0.01 & -0.02 & -0.48 & -0.05 & - & - \\
H11 & ref. & 1.75 & 1.56 & 5.63 & -0.58 & - & - \\
& calc. & 1.77 & 1.49 & 5.26 & -0.48 & - & - \\
& $\Delta$ & -0.01 & 0.07 & 0.36 & -0.11 & - & - \\
H12 & ref. & 2.28 & 4.10 & 3.21 & 0.33 & -0.69 & 1.46 \\
& calc. & 2.01 & 3.80 & 2.70 & 0.40 & -0.41 & 1.35 \\
& $\Delta$ & 0.27 & 0.30 & 0.51 & -0.07 & -0.28 & 0.11 \\
\hline
\end{tabular}


Table S2 Benzene @ 110 K. First row: TLS+ONIOM results; second row: mass adjusted multitemperature neutron reference results (Bürgi et al., 2002); third row: differences.

\begin{tabular}{rcrrrrrr}
\hline & & $U_{11}$ & $U_{22}$ & $U_{33}$ & $U_{12}$ & $U_{13}$ & $U_{23}$ \\
\hline $\mathrm{H} 1$ & ref. & 4.94 & 2.56 & 5.50 & 0.93 & 0.29 & 0.12 \\
& calc. & 4.58 & 2.74 & 5.15 & 0.96 & 0.02 & 0.00 \\
& $\Delta$ & 0.36 & -0.18 & 0.35 & -0.03 & 0.27 & 0.12 \\
$\mathrm{H} 2$ & ref. & 4.13 & 4.95 & 4.71 & 0.87 & 1.95 & -0.32 \\
& calc. & 4.12 & 5.00 & 4.69 & 0.78 & 2.20 & -0.46 \\
& $\Delta$ & 0.01 & -0.05 & 0.02 & 0.09 & -0.25 & 0.14 \\
$\mathrm{H} 3$ & ref. & 4.86 & 4.00 & 4.45 & -0.57 & 1.19 & 1.35 \\
& calc. & 4.47 & 4.18 & 4.05 & -0.78 & 1.03 & 1.28 \\
& $\Delta$ & 0.39 & -0.18 & 0.40 & 0.21 & 0.16 & 0.07 \\
\hline
\end{tabular}


Table S3 $\alpha$-glycine @ 23 K. First row: TLS+ONIOM results; second row: TLS + spectroscopy reference results (Destro et al., 2000); third row: differences.

\begin{tabular}{lccccccc}
\hline & & $U_{11}$ & $U_{22}$ & $U_{33}$ & $U_{12}$ & $U_{13}$ & $U_{23}$ \\
\hline $\mathrm{H} 1$ & ref. & 2.33 & 2.35 & 1.14 & 0.26 & 0.79 & 0.16 \\
& calc. & 2.33 & 3.77 & 1.16 & 0.70 & 0.95 & 0.43 \\
& $\Delta$ & 0.00 & -1.42 & -0.02 & -0.44 & -0.16 & -0.27 \\
$\mathrm{H} 2$ & ref. & 1.01 & 1.95 & 1.53 & -0.16 & 0.43 & -0.16 \\
& calc. & 1.28 & 2.03 & 1.63 & -0.11 & 0.24 & -0.14 \\
& $\Delta$ & -0.26 & -0.08 & -0.10 & -0.05 & 0.19 & -0.03 \\
$\mathrm{H} 3$ & ref. & 1.80 & 1.22 & 1.78 & 0.14 & 0.80 & 0.02 \\
& calc. & 2.21 & 1.34 & 2.39 & 0.13 & 0.78 & 0.09 \\
& $\Delta$ & -0.40 & -0.12 & -0.61 & 0.01 & 0.02 & -0.07 \\
$\mathrm{H} 4$ & ref. & 2.78 & 1.25 & 2.25 & 0.24 & 1.32 & 0.35 \\
& calc. & 2.62 & 1.08 & 2.57 & 0.16 & 1.27 & 0.51 \\
& $\Delta$ & 0.16 & 0.17 & -0.32 & 0.08 & 0.04 & -0.16 \\
$\mathrm{H} 5$ & ref. & 0.90 & 2.83 & 1.49 & -0.16 & 0.24 & -0.40 \\
& calc. & 1.17 & 2.76 & 1.52 & -0.18 & 0.13 & -0.47 \\
& $\Delta$ & -0.27 & 0.07 & -0.04 & 0.03 & 0.11 & 0.07 \\
\hline
\end{tabular}


Table S4 Xylitol @ 123 K. First row: TLS+ONIOM results; second row: adjusted neutron reference results (Madsen et al., 2003; Madsen et al., 2004); third row: differences.

\begin{tabular}{|c|c|c|c|c|c|c|c|}
\hline & & $U_{11}$ & $U_{22}$ & $U_{33}$ & $U_{12}$ & $U_{13}$ & $U_{23}$ \\
\hline \multirow[t]{3}{*}{$\mathrm{H} 1 \mathrm{~A}$} & ref. & 3.19 & 2.73 & 2.59 & 0.77 & -0.23 & -1.08 \\
\hline & calc. & 2.81 & 2.69 & 2.19 & 0.46 & -0.07 & -0.67 \\
\hline & $\Delta$ & 0.38 & 0.03 & 0.40 & 0.32 & -0.16 & -0.41 \\
\hline \multirow[t]{3}{*}{ H1B } & ref. & 1.93 & 3.93 & 2.77 & -0.68 & 0.17 & 1.17 \\
\hline & calc. & 1.92 & 3.27 & 2.67 & -0.48 & 0.20 & 0.89 \\
\hline & $\Delta$ & 0.01 & 0.66 & 0.10 & -0.19 & -0.03 & 0.29 \\
\hline \multirow[t]{3}{*}{$\mathrm{H} 2$} & ref. & 1.99 & 2.04 & 2.60 & 0.12 & 0.11 & -0.70 \\
\hline & calc. & 2.33 & 1.97 & 2.05 & 0.17 & 0.03 & -0.50 \\
\hline & $\Delta$ & -0.34 & 0.07 & 0.55 & -0.05 & 0.08 & -0.20 \\
\hline \multirow[t]{3}{*}{ H3 } & ref. & 2.33 & 1.98 & 2.21 & 0.22 & -0.04 & -0.64 \\
\hline & calc. & 2.35 & 2.10 & 2.17 & 0.20 & 0.02 & -0.67 \\
\hline & $\Delta$ & -0.02 & -0.12 & 0.04 & 0.02 & -0.05 & 0.03 \\
\hline \multirow[t]{3}{*}{$\mathrm{H} 4$} & ref. & 2.37 & 2.85 & 1.59 & -0.04 & -0.07 & 0.45 \\
\hline & calc. & 2.26 & 2.58 & 1.55 & -0.01 & 0.03 & 0.23 \\
\hline & $\Delta$ & 0.11 & 0.27 & 0.04 & -0.03 & -0.11 & 0.23 \\
\hline \multirow[t]{3}{*}{ H5A } & ref. & 2.46 & 2.66 & 4.64 & 0.23 & 0.36 & -1.74 \\
\hline & calc. & 2.35 & 2.51 & 2.94 & 0.26 & 0.12 & -1.08 \\
\hline & $\Delta$ & 0.12 & 0.15 & 1.71 & -0.03 & 0.24 & -0.66 \\
\hline \multirow[t]{3}{*}{ H5B } & ref. & 2.35 & 3.21 & 2.79 & -0.01 & -0.51 & 1.07 \\
\hline & calc. & 2.42 & 3.01 & 1.94 & 0.17 & -0.15 & 0.32 \\
\hline & $\Delta$ & -0.07 & 0.20 & 0.85 & -0.18 & -0.35 & 0.75 \\
\hline \multirow[t]{3}{*}{ H11 } & ref. & 1.70 & 3.15 & 2.10 & -0.12 & 0.43 & 0.32 \\
\hline & calc. & 1.90 & 3.71 & 2.24 & 0.14 & 0.46 & 0.15 \\
\hline & $\Delta$ & -0.20 & -0.56 & -0.14 & -0.26 & -0.03 & 0.17 \\
\hline \multirow[t]{3}{*}{ H12 } & ref. & 2.70 & 3.00 & 2.17 & -0.46 & 0.68 & -0.19 \\
\hline & calc. & 2.94 & 3.31 & 1.71 & -0.60 & 0.76 & -0.20 \\
\hline & $\Delta$ & -0.24 & -0.30 & 0.46 & 0.15 & -0.07 & 0.01 \\
\hline \multirow[t]{3}{*}{ H13 } & ref. & 3.01 & 1.79 & 2.24 & -0.10 & 0.10 & 0.21 \\
\hline & calc. & 4.27 & 1.75 & 2.70 & -0.27 & -0.23 & 0.24 \\
\hline & $\Delta$ & -1.25 & 0.03 & -0.46 & 0.17 & 0.33 & -0.02 \\
\hline \multirow[t]{3}{*}{ H14 } & ref. & 3.17 & 1.97 & 2.73 & 0.82 & -0.28 & 0.13 \\
\hline & calc. & 3.46 & 2.03 & 2.35 & 0.83 & -0.62 & 0.22 \\
\hline & $\Delta$ & -0.30 & -0.06 & 0.38 & -0.01 & 0.33 & -0.09 \\
\hline \multirow[t]{3}{*}{ H15 } & ref. & 2.61 & 2.77 & 2.23 & -0.62 & 0.02 & -0.68 \\
\hline & calc. & 2.82 & 3.31 & 2.53 & -0.82 & -0.29 & -0.71 \\
\hline & $\Delta$ & -0.21 & -0.53 & -0.30 & 0.20 & 0.32 & 0.03 \\
\hline
\end{tabular}


Table S5 MNA @ 100 K. First row: TLS+ONIOM results; second row: adjusted neutron reference results (Whitten, Turner et al., 2006); third row: differences.

\begin{tabular}{|c|c|c|c|c|c|c|c|}
\hline & & $U_{11}$ & $U_{22}$ & $U_{33}$ & $U_{12}$ & $U_{13}$ & $U_{23}$ \\
\hline \multirow[t]{3}{*}{ HNA } & ref. & 2.55 & 2.46 & 3.98 & -0.19 & -0.69 & 0.18 \\
\hline & calc. & 2.02 & 3.08 & 5.47 & -0.36 & -0.89 & -0.43 \\
\hline & $\Delta$ & 0.53 & -0.62 & -1.49 & 0.16 & 0.20 & 0.61 \\
\hline \multirow[t]{3}{*}{ (NB } & ref. & 3.06 & 1.95 & 4.95 & 0.33 & -0.84 & 0.51 \\
\hline & calc. & 2.92 & 2.15 & 5.19 & 0.40 & -0.54 & 0.72 \\
\hline & $\Delta$ & 0.13 & -0.19 & -0.24 & -0.07 & -0.30 & -0.21 \\
\hline \multirow[t]{3}{*}{ H3 } & ref. & 2.59 & 1.53 & 3.95 & 0.30 & -0.81 & 0.09 \\
\hline & calc. & 2.91 & 1.67 & 4.08 & 0.16 & -0.74 & 0.27 \\
\hline & $\Delta$ & -0.31 & -0.14 & -0.14 & 0.14 & -0.07 & -0.17 \\
\hline \multirow[t]{3}{*}{ H5 } & ref. & 2.05 & 1.96 & 4.12 & -0.40 & -0.72 & 0.16 \\
\hline & calc. & 2.29 & 2.35 & 4.20 & -0.61 & -0.87 & -0.12 \\
\hline & $\Delta$ & -0.24 & -0.39 & -0.08 & 0.21 & 0.15 & 0.28 \\
\hline \multirow[t]{3}{*}{ H6 } & ref. & 2.85 & 1.79 & 4.55 & 0.09 & -0.25 & 0.33 \\
\hline & calc. & 2.97 & 1.66 & 4.59 & 0.15 & -0.81 & 0.24 \\
\hline & $\Delta$ & -0.13 & 0.13 & -0.03 & -0.06 & 0.56 & 0.09 \\
\hline \multirow[t]{3}{*}{ H7A } & ref. & 3.75 & 3.76 & 2.56 & -0.78 & -0.84 & 0.12 \\
\hline & calc. & 3.77 & 4.13 & 2.48 & -0.80 & -1.06 & 0.11 \\
\hline & $\Delta$ & -0.02 & -0.37 & 0.08 & 0.01 & 0.22 & 0.01 \\
\hline \multirow[t]{3}{*}{ H7B } & ref. & 2.44 & 4.46 & 4.06 & -0.82 & 0.50 & -0.33 \\
\hline & calc. & 2.19 & 4.37 & 4.20 & -0.69 & 0.55 & -0.42 \\
\hline & $\Delta$ & 0.25 & 0.10 & -0.14 & -0.13 & -0.06 & 0.10 \\
\hline \multirow[t]{3}{*}{$\mathrm{H} 7 \mathrm{C}$} & ref. & 3.53 & 1.94 & 5.98 & 0.01 & -1.31 & 0.24 \\
\hline & calc. & 3.62 & 1.94 & 5.22 & -0.14 & -1.02 & 0.17 \\
\hline & $\Delta$ & -0.09 & 0.01 & 0.76 & 0.16 & -0.29 & 0.07 \\
\hline
\end{tabular}

